\title{
Analysis of fungal dynamic changes in the natural fermentation broth of 'Kyoho' grapes based on high- throughput sequencing
}

\section{Wenjuan Zhao}

Southwest Forestry University

\section{Zhiming Zhang}

Southwest Forestry University

\section{Yuhong Gao}

Southwest Forestry University

Xiaozhen Liu

Southwest Forestry University

Chaojin Du

Southwest Forestry University

\section{Fuxian Ma}

Southwest Forestry University

\section{Sujie Wang}

Southwest Forestry University

Wanyuan Shi

Southwest Forestry University

\section{Yanping Yang}

Southwest Forestry University

\section{Ruyou Deng}

Southwest Forestry University

Hanyao Zhang ( $\square$ zhanghanyao@hotmail.com )

Southwest Forestry University https://orcid.org/0000-0001-7440-4348

\section{Research Article}

Keywords: 'Kyoho' grape, natural fermentation, high-throughput sequencing, dynamics diversity, pathways, dominant species.

Posted Date: February 24th, 2022

DOI: https://doi.org/10.21203/rs.3.rs-1381008/v1 
License: (c) (i) This work is licensed under a Creative Commons Attribution 4.0 International License. Read Full License 


\section{Abstract}

The 'Kyoho' grape (Vitaceae, Plantae) has large ears, plenty of flesh, and rich nutrition, and is planted across a large area in China. There are few reports on this variety in winemaking, especially on the dynamic changes of fungi in the wine fermentation broth. In this study, we used the 'Kyoho' grapes as raw materials and adopted a high throughput to analyze dynamic changes in fungal species composition of the natural fermentation broth at four time points: day one (D1P), day three (D3P), day five (D5P), and day fifteen (D15P). Changes in fungal metabolic pathways and the dynamic changes of dominant yeast were also analyzed. A total of 78 families, 110 genera, and 137 species were detected, in the natural fermentation broth samples. The control check (CK) had 49 families, 60 genera, and 72 species. A total of 66 differential metabolic pathways were enriched; of those 66,41 pathways were up-regulated compared to CK, such as pyrimidine deoxyribonucleotides biosynthesis from CTP (PWY 7210), CDP-diacylglycerol biosynthesis I (PWY 5667), and chitin degradation to ethanol (PWY 7118). Changes in fungal metabolic pathways were in line with the dynamic changes of dominant yeast species in the whole process of fermentation. Pichia kluyveri , P. membranifaciens, and Citeromyces matritensis are the dominant species in the later stages of natural fermentation. These yeast species may play vital roles in the 'Kyoho' wine industry in the future.

\section{Introduction}

The 'Kyoho' grape is a dicotyledonous plant belonging to the Vitaceae and the genus Vitis ${ }^{[1]}$. It is one of the main grape varieties planted in China, with obvious characteristics such as large ears, large grain, good resistance, and strong adaptability ${ }^{[2]}$. The fruit grains become red and blackish purple while the fruit is ripe; the flesh is tight and juicy. Its taste is sweet and sour, and the fruit contains sugar more than $15 \%$, acidity less than $0.5 \%$, vitamin C $4 \mathrm{mg} / \mathrm{g}$, and total soluble solids $130 \mathrm{mg} / \mathrm{g}^{[3]}$. Studies have shown that the 'Kyoho' grape has a higher aroma compared with other grapes, and the C6 aldehyde makes it have good aroma quality ${ }^{[4]}$. Zhen et al. took the 'Kyoho' grape as raw material and clarified that fertilizer supply was in direct proportion with total aroma, and 'Kyoho' had a high content of fatty aldehyde and fatty acid ${ }^{[5]}$. Zhou et al. used 'Kyoho' grape concentrate to make wine and found the organic acid content was the same as that of the traditional process, and the physical and chemical properties were in line with national standards ${ }^{[6]}$. Huang et al. conducted a comprehensive evaluation of the wine quality after fermentation of the 'Kyoho', which indicated that the 'Kyoho' grape is a variety for fresh food and winemaking ${ }^{[7]}$. In addition, Guan et al. mixed the juice of Myrtle and 'Kyoho' for fermentation ${ }^{[8]}$. Jia et al. isolated some yeast strains from the fermentation broth of 'Kyoho' grapes with higher sugar tolerance, acid tolerance, and alcohol tolerance than Saccharomyces cerevisiae ${ }^{[9]}$. From the current point of view, most of the research focused on 'Kyoho' grapes cultivation, fruit quality improvement, fruit storage, and preservation. There are few reports on wine brewing, the separation of brewing yeast, and the fungal diversity after fermentation of this variety. 
High throughput sequencing technology enables detailed, comprehensive analysis of the transcriptome and genome data of a species. Therefore, this technology is also called deep sequencing or nextgeneration sequencing (NGS) ${ }^{[10,11]}$. This technique has a quantitative function, which could determine the abundance of a species component in the sample. In addition, the cost of using this technique is lower, and it could work completed faster, better than ITS PCR and fluorescent ITS PCR capillary electrophoresis ${ }^{[12]}$. At present, high-throughput sequencing technology is widely used in analyzing the microbial composition of liquor brewing, the diversity of the microbial community in the fermented soybean Liquid, the fungal diversity, and the microbial community structure of the traditional fermented Kohlrabi analysis, analysis of microbial community diversity in the fermented vegetable juice, and so

on ${ }^{[11-15]}$. High-throughput sequencing technology has been widely used in microbial diversity sequencing, microbial community structure sequencing, dynamic changes of dominant species, etc. [16]. In addition, the application of this technique in microbial community dynamic analysis has been relatively mature. It also can be used to comprehensively understand the molecular evolution, species composition, and gene regulation of a species ${ }^{[17]}$.

In this study, we adopted high-throughput sequencing to analyze changes in fungal species' composition, fungal metabolic pathways, and the dynamic changes of dominant yeast in the fermentation broth of 'Kyoho' grapes under natural fermentation, different periods, and enrichment medium cultivation. This study provides a reference for the study of microbial diversity in the 'Kyoho' grape broth and a scientific basis in 'Kyoho' grape wine brewing.

\section{Materials And Methods}

\subsection{Materials}

Grapes picked in Anning, Kunming, Yunnan Province, China were ripe, plump, and free of diseases.

\subsection{Natural fermentation of 'Kyoho' grapes}

We weighed $500 \mathrm{~g}$ of the grapes and transferred them to an ultra-clean workbench. After removing any broken grapes, we evenly placed them in three one-liter sterile conical flasks at 28 ${ }^{0} \mathrm{C}$ for fermentation. We took two milliliters of broth and placed them in a centrifuge tube at four-time points, one, three, five, and 15 days, and named them D1P, D3P, D5P, and D15P, respectively. The samples were centrifuged (6000 rpm, $10 \mathrm{~min}$ ) with PBS buffer in a centrifuge three times, then cell pellets obtained were stored in liquid nitrogen $\left(-80^{\circ} \mathrm{C}\right)$ for high-throughput sequencing.

\section{3 'Kyoho' microbiota grown on YPD}


We weighed $50 \mathrm{~g}$ of the grapes and transferred them into three one-liter sterile conical flasks containing $167 \mathrm{~mL}$ YPD liquid medium, cultured at a constant temperature $\left(28^{\circ} \mathrm{C}\right)$, named control check (CK). After a culture time of three days, the fermentation broth became turbid, and three replicated samples of two milliliters each were taken from the fermentation broths. After centrifuging $(6000 \mathrm{rpm}$, $10 \mathrm{~min}$ ), the microbial samples were rinsed with PBS buffer three times, then stored in liquid nitrogen (-80 $\left.{ }^{0} \mathrm{C}\right)$ for high-throughput sequencing.

\subsection{High-throughput sequencing and analysis}

Total DNA was extracted from each microbial sample using the CTAB method ${ }^{[18]}$. High-throughput sequencing was completed by Baiqi Biotechnology Co, Ltd. (Hubei Province, China). The metagenomics sequencing was conducted, and ITS5: GGAAGTAAAAGTCGTAACAAGG and

ITS2: GCTGCGTTCTTCATCGATGC were used for sequencing ${ }^{[19]}$. The sequencing data were analyzed on the Genescloud platform (www.genescloud.cn).

\subsubsection{Sequence processing and analysis}

Paired-end sequencing of DNA fragments was performed on the Illumina platform. Vsearch (v2.13.4linux-x86_64) and cutadapt (v2.3) were employed to denoise and cluster the sequences ${ }^{[20,21]}$. After using the qiime cutadapt trim-paired to excise sequence primer fragments, the sequences unmatched with primers were discarded. The Vsearch module was used for splicing, deduplicating, and dechimericing the sequences, and the UNITE database (Release 8.0, https://unite.ut.ee/) was used to filter the concentrated chimeras to obtain high-quality ones. The QIIME2 (classify-sklearn algorithm https://github.com/QIIME2/q2-feature-classifier) was employed to annotate the characteristic sequences of each Operational Taxonomic Units (OTUs) in the Naive Bayes classifiers ${ }^{[22,23]}$. The QIIME2 qiime feature-table Rarefy function was employed to set the leveling depth to $95 \%$ of the smallest sample sequence size, and the final OTUs were obtained.

\subsubsection{Species composition analysis}

The number of OTUs and taxa were counted based on the result of the taxonomic annotations of sequences. The QIIME2 software was used to draw a pie chart according to the method described by Li et al. ${ }^{24]}$. Using $\mathrm{R}$ language, graph, and ggplot2 package, a microbial classification hierarchy tree was drawn, and the abundance value of each OTU group was added to the pie chart for species composition analysis. Through the coloring of OTU dots, the taxonomic composition of microorganisms was emphasized according to the method adopted by Ondov et al. ${ }^{[25]}$. Krona software (https://github.com/marbl/Krona/wiki) was used to display the interaction of its taxonomic composition. Heat maps for species composition analysis were drawn, using $\mathrm{R}$ language and heat map software. 


\subsubsection{Alpha diversity analysis}

Alpha diversity refers to the diversity within a sample. Commonly used alpha diversity indices are Chao1, Good's coverage, Observed species, Pielou's evenness, Shannon, Simpson, etc. ${ }^{[26-31]}$. The Chao 1 index measures species richness and is used to estimate the number of species in a sample ${ }^{[26]}$. Good's coverage refers to the coverage rate of each sample library, and this index reflects whether the sequencing result represents the real situation of the microorganisms in the sample ${ }^{[27]}$. The Observed species index represents the number of species contained in the samples ${ }^{[28]}$. Pielou's evenness indicates the uniformity of the community ${ }^{[29]}$. The Shannon index combines abundance and evenness, giving more weight to rare species ${ }^{[30]}$. The Simpson index also combines abundance and evenness but focuses more on common species ${ }^{[31]}$.

QIIME2, R language, and ggplot2 package were employed for alpha diversity analysis ${ }^{\text {[26-31]. After }}$ using the unleveled OTU table, calling the "qiime diversity alpha-rarefaction" command, and setting the minimum leveling depth to ten and the minimum sequencing depth to $95 \%$ of the sample sequence, each depth value was flattened ten times to calculate the alpha diversity index.

\subsubsection{Beta diversity analysis}

Beta diversity refers to the differences between samples or groups and is often used to analyze whether the differences in microbial composition between two groups are significant. Commonly used beta diversity indices are Jaccard, Bray Curtis, unweighted UniFrac, and weighted UniFrac ${ }^{[32-34]}$. The Jaccard

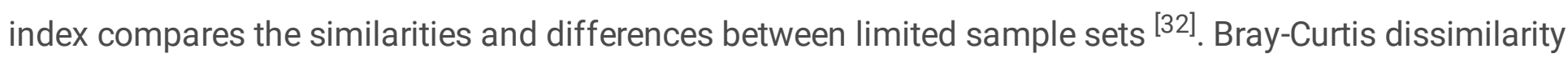
is a measure used to analyze the difference in species composition in different places ${ }^{[33]}$. Unweighted UniFrac can detect the presence of variations between samples, while weighted UniFrac can further quantitatively detect the variation between samples on different lineages ${ }^{[34]}$.

The QIIME2 was employed to analyze the leveled OTU table ${ }^{[35]}$. The "qiime diversity core metrics phylogenetic" or "qiime diversity core metrics" command was used to calculate four distance matrices such as Jaccard, Bray Curtis, unweighted UniFrac, and weighted UniFrac or two distance matrices such as Jaccard and Bray Curtis. Then PCoA analysis based on these distance matrices was performed.

In Beta diversity clustering analysis, the hierarchical clustering analysis method was conducted ${ }^{[36]}$. Using the cluster analysis of the Bray-Curtis distance matrix, the R language, Vegan, APE, and ggtree package were employed to analyze the differences among the samples.

\subsubsection{Function prediction}

PICRUSt2 was used to predict sample function abundance using the rrs gene encoding bacterial $16 \mathrm{~S}$ rRNA as a maker ${ }^{[37]}$. According to the analysis processes on the PICRUSt2 website 
(https://github.com/picrust/picrust2/wiki), the metabolic pathway abundance and functional unit files were normalized. According to the metabolic pathway database and calculation methods, the abundance values and the second-level pathways were calculated based on the functional units obtained. The $R$ language, metagenomeSeq package tool, and normalized pathway/group abundance values were used to calculate the different significant degrees. Finally, we used the $\mathrm{R}$ language to draw a histogram of the species composition of the functional microbe in the metabolic pathway and analyze the data in the abundance table.

\section{Results And Analysis}

\subsection{Analysis of sample sequencing data}

The numbers of original sequences corresponding to the D1P, D3P, D5P, D15P, and CK obtained were $139,893,132,962,142,367,122,298$, and 123,262, respectively. After slicing and filtering, the effective sequences were 123952, 109471, 123695, 108724, and 109861, and the average length was $232 \mathrm{bp}$. According to the $97 \%$ similarity, the flattened samples obtained 305 OTUs.

\subsection{Analysis of species composition}

Through high-throughput sequencing analysis, a total of 78 families, 110 genera, and 137 species were detected. The classification detail is in Supplemental Table 1. Figure 1 shows the tree circle accumulation obtained from these samples.

There were a total of five categories. Among them, the yeast categories had more species, and the accumulation circle was relatively large, while three accumulation circles contained fewer species, and the accumulation circle was also small.

The Krona pie chart showed the classification level and abundance information of the species in the sample ${ }^{[24]}$. The results of the Krona analysis are shown in Figure 2. The sequencing results showed that the yeasts accounted for $64 \%$ of the total sequences, of which the Citeromyces genus accounted for $42 \%$, and it was a dominant genus. But the other types of fungi were relatively rare.

Based on the analysis of species composition at the taxonomic level, the results are shown in Figure 3 . Mycosphaerella tassiana had the largest proportion (35.22\%), followed by others (22.49\%), in the D1P samples. Torulaspora delbrueckii accounted for $51.31 \%$, followed by Citeromyces matritensis with $38.45 \%$, among the D3P samples. In the D5P ones, T. delbrueckii and C. matritensis accounted for $79.67 \%$ and $19.62 \%$, respectively. In the D15P samples, T. delbrueckii accounted for $62.91 \%$. C. matritensis accounted for $22.64 \%$.

The content of Debaryomyces prosopidis in CK was relatively higher, accounting for $61.45 \%$ of the samples. Compared with D1P, at the level of species classification, there were fewer fungi in CK than D1P, 
and the composition of bacteria in the two samples was significantly different (see Figure 3).

The results of the heat map analysis are in Figure 4. At the level of species classification, fungi were more abundant in sample D1P. During the natural fermentation process, the amount of $T$. delbrueckii gradually increased, reached largest in D5P, then decreased in D15P. The abundances of $C$. matritensis, Hanseniapora nectarophila, and Hyphopichia burtonii were the largest in D3P, and then decreased, $C$. matritensis showed an increasing trend at D15P. The amounts of Aureobasidium pullulans, Pichia membranifaciens, P. kluyveri, and Zygosaccharomyces bailii in D1P were the most and then decreased. The abundances of $P$. membranifaciens and $P$. kluyveri increased at D15P. Comparing the results of CK with D1P, the $P$. membranifaciens, $M$. tassiana, and Z. bailii decreased. However, the amounts of $D$. prosopidis, P. kluyveri, and $P$. terricola increased in CK.

\subsection{Alpha diversity index analysis}

The results of the Alpha diversity index analysis are in Table 1. The ratio of Shannon index to Simpson index of D1P was higher than that of $\mathrm{CK}$, and the D1P species was rich. The ratio of these two indexes of D5P was smaller, and the abundance of D5P was smaller than that of D1P. The index ratio of D15P was larger than that of D5P. In short, in the samples of natural fermentation, the ratio of Shannon index to Simpson index showed a trend of first decreasing and then increasing. Pielou's evenness index affected the number of species in the sample and the uniformity of individual distribution. Compared the Pielou's evenness index of the naturally fermented with CK, the value of CK was smaller. It indicated that the microbial community was not stable under the condition of enrichment culture, and the community in the

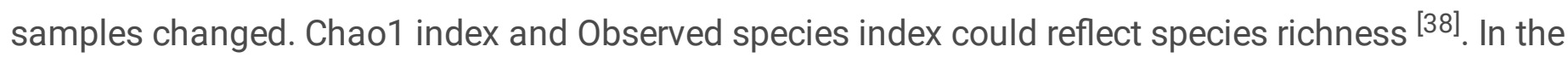
natural fermentation samples of 'Kyoho' grapes, both indexes of D1P were larger than other samples, followed by D3P, and, starting from D3P, the two indexes began to decrease, and when it came to D15P, the two indexes were higher than D5P. The specific changes of the index are in Figure 5.

\subsection{Beta diversity analysis}

The top ten species were selected from the samples for cluster analysis (see Figure 6). The samples were divided into two branches: natural fermentation and enrichment culture. The D1P samples were the furthest away from the other ones. It indicated that the species composition between D1P and other samples was quite different. The tree distances between D3P, D5P, and D15P were relatively close. It indicated that the fungi composition of these three samples was similar. One was far away from the other two D15P samples, and the species compositions were quite different among these three samples. The species composition of D1P was relatively rich, and the species composition of CK was single. $Z$. bailii showed a decreasing trend with the increase of fermentation time, T. delbrueckii and $C$. matritensis also showed dynamic changes with the change of fermentation time. The proportion of $D$. prosopidis and Fomes fomentarius of $\mathrm{CK}$ were larger than those in natural fermentation samples. 


\subsection{Function prediction}

In this study, a total of five first-level functional pathways and 29 second-level functional pathways were involved in the five samples. For the statistics, please see Supplementary Figure 1, and for the value, please refer to Supplemental Table 2. The differences of the sample abundance values were calculated, and the results are in Supplementary Figure 2. Fatty Acid and Lipid Biosynthesis (No.5), Carbohydrate Degradation (No.10), Fatty Acid and Lipid Degradation (No.12), and Glycan Biosynthesis (No.24) functional Pathways were higher in enriched culture samples than naturally fermented samples. In general, 13 pathways constantly changed during the fermentation process.

To explore the reasons for the changes in functional pathways, we had made statistics on the pathways involved in the metabolism of 'Kyoho' grapes. For the details, please see Supplemental Table 3. In this study, 66 differential metabolic pathways were involved, of which 41 up-regulated and 27 downregulated. The difference value of down-regulation pathways was greater than that of the upregulation ones. Five metabolic pathways with significant up-regulation and down-regulation were screened out, respectively, as shown in Supplementary Figure 3.

To further explore the reasons for changes in pathways, the species composition of these top ten positively and negatively regulated metabolic pathways were analyzed, as was the species composition of different pathways. See Supplemental Table 4 for details. The statistical table showed that the microbial species composition of the phospholipases (LIPASYN PWY) and gluconeogenesis I (GLUCONEO PWY) metabolic pathways was quite different, and there were almost no similar microbial species in other ones. Cutaneotrichosporon curvatus only appeared in the yeast, monoacylglycerol metabolism (PWY 7420), phospholipid remodeling (PWY 7409), glucose, and glucose-1-phosphate degradation (GLUCOSE1PMETAB PWY), and chitin degradation to ethanol (PWY 7118) metabolic pathways. The expression changes of this species in these metabolic pathways are in Supplementary Figure 4.

The common species in the five metabolic pathways of pyrimidine deoxyribonucleotides biosynthesis from CTP (PWY 7210), CDP-diacylglycerol biosynthesis I (PWY 5667), chitin degradation to ethanol (PWY 7118), the super pathway of phosphatidate biosynthesis (PWY 7411), and 1,3-propanediol biosynthesis (PWY 7385) were T. delbrueckii, P. kluyveri, D. prosopidis, H. burtonii, Aureobasidium pullulanas, and $A$. medicichiensis. See Supplementary Figure 4 for the changes of microbial species in the pathway. During the natural fermentation processing, the amounts of $T$. delbrueckii and $C$. matritensis increased first and then decreased in these five metabolic pathways, which was 0 in CK. The amount of $H$. burtonii reached the maximum in D3P samples, then gradually decreased in the D5P and D15P samples. This species did not appear in CK either. The abundance value of $P$. kluyveri was 0 in D1P samples, increased in D3P ones, decreased in D5P ones, rose in D15P ones, and had a larger value in CK. The abundance value of $P$. membranifaciens in D1P samples was larger, then decreased in D3P and D5P, then increased in D15P. The abundance value of CK was similar to that of D15P ones. D. prosopidis 
and $A$. pullulans showed a decreasing trend in natural fermentation samples as the fermentation time increased. The abundance value in CK was similar to the abundance value in D1P.

\section{Discussion}

Generally, the greater the ratio of Shannon index to Simpson index, the higher the diversity, and vice versa, the lower the species diversity was ${ }^{[39]}$. In this study, the index ratio of D1P was larger than that of CK, but the species number was less than CK. It may be due to errors in the sequencing. In the processes of natural fermentation, the abundance of fungi decreased first and then rose with the increase of time, which was the same as the results of former studies ${ }^{[40]}$. As the fermentation time increases, the alcohol content in the fermentation environment increases, the organic matter content decreases, and toxic metabolites are produced, so the microbes in the fermentation environment decrease, the growth of some non-Saccharomyces yeast are inhibited, and the tolerant microbe continues to grow ${ }^{[41,42]}$. The YPD medium was used as a fungus-enriched medium, and there were more fungi than natural fermentation. It may be because the peptone in the YPD medium provides carbon and nitrogen sources, the yeast extract provides $\mathrm{B}$ vitamins, and glucose provides energy materials, which makes the growth of fungi more vigorous ${ }^{[43]}$. The species composition heat map defines the value of each variable, which is convenient for the analysis of the sample species composition, and the similarity and difference of the values are judged by the color gradient change and the similarity.

In this research, the content of $C$. matritensis and $H$. burtonii reached the most in D3P ones. It was similar to the results that Wang et al. Reported ${ }^{[44]}$. Both T. delbrueckii and C. matritensis have ethanol fermentability, and when there are sulfides in the fermentation broth, the growth of $T$. delbrueckii will be inhibited. The account of $H$. burtonii reached its maximum value in D3P and then decreased. It may be due to its excessive sensitivity to alcohol ${ }^{[45]}$. Čadež et al. also pointed out that non-Saccharomyces yeast is restricted during the 3-4 days of fermentation, and some specific non-Saccharomyces yeast will continue to exist until the fermentation is completed ${ }^{[46]}$. H. nectarophila was a yeast isolated from flowers ${ }^{[46]}$. The study on the performance of this species is rare. In this study, the abundance value of this species first increased and then decreased with the increase of fermentation time. Analysis of species involved in metabolism revealed that $C$. curvatus, as an oleaginous yeast, can use different renewable carbon sources to produce lipids ${ }^{[47,48]}$.

Brežná et al. used fluorescent ITS PCR and capillary electrophoresis to study the bacterial diversity of Frankovka (red wine) and Veltlin (white wine) and pointed out that using the f-ITS PCR study on the diversity of microbial communities in wine has advantages such as being simple and economical; however, its identification of strains can result in amplicons of the same or very similar length, or ambiguous identification of certain amplicons ${ }^{[49]}$. Park et al. used morphological, physiological, and phylogenetic analysis; polymerase chain reaction (PCR); and PCR-mediated denaturing gradient gel 
electrophoresis (DGGE). The microbial diversity of Fujian starter and traditional Korean starter was compared. It was found that the diversities of bacteria in the two starters were quite different, and it was pointed out that there was a big difference between the two starters ${ }^{[50]}$. Xie et al. used high-throughput sequencing technology to analyze the chemical metabolism of fungi in "Lianzhifan Solution" traditional Chinese medicine fermentation broth and screened out key strains in traditional Chinese medicine preparations ${ }^{[51]}$. However, there are no reports on using high-throughput sequencing technology to study the fungal dynamic changes in the natural fermentation broth of grape wine.

In this study, we identified a large number of wine-related yeasts from the natural fermentation broth of 'Kyoho' grape by high-throughput sequencing technology, such as Hanseniaspora vineae, T. delbrueckii, $Z$. bailii, P. membranifaciens, P. terricola, Meyerozyma guilliermondii, and other non-Saccharomyces yeast. These non-Saccharomyces yeasts, with their unique metabolic pathways and strong enzymatic activity, reduce alcohol production during the winemaking process while increasing glycerol, terpene, and ester content, releasing mannose proteins or polysaccharides to improve taste and enhance color stability ${ }^{[52]}$. These bacteria can participate in the hydrolysis of aromatic compound precursors, ester synthesis, alcohols, glycerol, and other flavoring substances during the winemaking process; they then naturally die out in the later stages of fermentation, with the $S$. cerevisiae eventually taking the lead in completing the fermentation [53]. Guarcello et al. cultured, isolated, and identified C. matritensis from "oil olive" extracts and found that this species tolerated alcohol and osmotic pressure well ${ }^{[54]}$.

Studies have shown that Pichia's esterase will grow slowly during one to four days of fermentation, and the number of yeasts will decrease after four days ${ }^{[55]}$. The behavior of the Pichia species in this study is different from the results of previous studies, which may be due to the inconsistency of the grape varieties used or the various species or strains involved. However, the presence of Pichia can improve the quality and aroma of wine and increase the lipid content during fermentation, and $P$. kluyveri can reach the peak of alcohol production within $48-72 \mathrm{~h}$ after fermentation ${ }^{[56,57]}$. D. prosopidis and A. pullulans decreased with time increased during natural fermentation. It may be due to the accumulation of organic acids, the decrease of $\mathrm{pH}$ value, and the consumption of nutrients, causing some fungi to be replaced by high-tolerant fungi or the direct decline of poorly tolerated fungi ${ }^{[54]}$. Through the analysis of fungal biodiversity in the natural fermentation broth of 'Kyoho' grapes, the dynamic changes of fungal diversity found will provide a basis for the development and utilization of non-Saccharomyces yeast and the development of synthetic biology and provide new potential applications in the field of biotechnology in the future.

\section{Conclusion}

This is the first report on using high-throughput sequencing technology to study the fungal dynamic changes in the natural fermentation broth. By comparing the species under natural fermentation conditions and enriched fermentation conditions, it was found that as the fermentation time increased, the species numbers detected in the samples increased first and then decreased. However, as time went 
on, the abundance of the species with poor tolerance to alcohol would decrease continually. Z. bailii, $D$. prosopidis, and $A$. pullulans were the most sensitive to the fermentation environment. These species were the most abundant species on the first day of fermentation. $H$. nectarophila, $C$. curvatus, and $H$. burtonii may have higher environmental and nutritional requirements. They were the dominant species on the third day, and then their amounts slowly decreased. T. delbrueckii was the dominant species of D5P samples. P. kluyveri, P. membranifaciens, and $C$. matritensis are the dominant species in the later stages of natural fermentation. These yeast species may play vital roles in the 'Kyoho' wine industry in the future.

\section{Abbreviations}

CK, Control check; D1P, Sample of day 1; D3P, Sample of day 3; D5P, Sample of day 5; D15P, Sample of day 15; GLUCONEO PWY, gluconeogenesis I; GLUCOSE1PMETAB PWY, Glucose, and glucose-1-phosphate degradation; LIPASYN PWY, Phospholipases; NGS, Next-generation sequencing; OTUs, Operational taxonomic units; PBS, Phosphate buffer saline; PWY 5667, Metabolic pathways of CDP-diacylglycerol biosynthesis I; PWY 7210, Metabolic pathways of pyrimidine deoxyribonucleotides biosynthesis from CTP; PWY 7118, Metabolic pathways of chitin degradation to ethanol; PWY 7420, Metabolic pathways of monoacylglycerol metabolism; PWY 7385, Metabolic pathways of 1,3-propanediol biosynthesis; PWY 7409, Metabolic pathways of phospholipid remodeling; PWY 7411, The super pathway of phosphatidate biosynthesis; YPD, Yeast extract peptone dextrose.

\section{Declarations}

\section{Ethics approval and consent to participate}

The experiment materials do not include a human being or animal. Hence, ethics approval and consent to participate are not applicable.

\section{Consent for Publication}

Not applicable.

\section{Availability of data and material}

All data generated or analyzed during this study are included in this published article. Metagenomics seq data were presented at the National Centerfor Biotechnology Information Data Center (accession number SRR16674728-SRR16674742).

\section{Competing interests}

The authors declare that they have no competing interests.

\section{Funding}


The project is funded by grants from the National Natural Science Foundation of China $(32160556,31760450)$ and the Joint Project of Agricultural Basic Research in Yunnan Province (2018FG001-038).

\section{Author contributions}

$\mathrm{WZ}, \mathrm{ZZ}$, and $\mathrm{YG}$ analyzed the experimental data and drafted the manuscript. $\mathrm{XL}, \mathrm{CD}$, and $\mathrm{FM}$ conducted the study. SW, WS, YY, and RD helped assess the experimental data. HZ conceived of the study and assisted in writing the manuscript. All authors agreed to the final version of the manuscript.

\section{References}

1. Xiu D R. (1990) Discussion on the current development of 'Kyoho' grape[J].Shanxi Fruit Tree. (in Chinese).

2. Chao W J. (2006) Pay attention to 'Kyoho' varieties and give full play to the advantages of 'Kyoho' varieties[J].Friends of Fruit Farmers. (in Chinese).

3. Liang L Y, NanL P, Yan S J. (2003) Effects of freshness keeping agent on respiratory intensity and storage quality of 'Red Globe', 'Kyoho' grapes[J].Transactions of the Chinese society of agricultural engineering. (in Chinese).

4. Zhou J M. (2013) Composition and metabolic regulation of aroma substances in table grapes[D].Shandong Agricultural University. (in Chinese).

5. Zhen H Y, Wang J, Li C W, et al. (2005) Study on the higher alcohols in fermentation of wine of 'Kyoho'[J].Sino-Overseas Grapevine \& Wine. (in Chinese).

6. Zhou G Q, Zhang G F. (2008) Fast grape wine production by 'Kyoho' condensed grape juice[J]. LiquorMaking Science \& Technology. (in Chinese).

7. Huang J L, LI Yang R, Bai X J, et al. (2011) Study on wine-making characteristics of 'Kyoho' grape with two-harvests per year in Guangxi[J].Liquor-Making Science \& Technology. (in Chinese).

8. Guan J X, Yang Y, Zhang J, et al. (2005) A method for making Myrtle 'Kyoho' wine: CN201510403030.1[P]. (in Chinese).

9. Jia C, Cao L, Ma A. (2016) Isolation of yeast strains from Kyoho grape and study on their tolerance[J]Liquor-Making Science \& Technology. https//:doi.10.13746/j.njkj.2016042.

10. Sultan M, Schulz MH, Richard $\mathrm{H}$, et al. (2008) A global view of gene activity and alternative splicing by deep sequencing of the human transcriptome[J]. Science. https//:doi.10.1126/science.1160342.

11. Margulies M, Egholm M, Altman W, et al. (2005) Genome sequencing in microfabricated high-density picolitre reactors[J]. Nature. https//:doi.10.1038/nature03959.

12. Wheeler D A, Srinivasan M, Egholm M, et al. (2008) The complete genome of an individual by massively parallel DNA sequencing[J]. Nature. https//:doi. 10.1038/nature06884.

13. Yin L B, Lei Z M, Zhang C Y, et al. (2017) Bacterial community structure and diversity in fermented waste water from soybean processing by high throughput sequencing technology[J]. China Food 
Additives. https//:doi. 10.3969/j.issn.1006-2513.2017.05.009. (in Chinese)

14. Wu X, Liu Q, Zhang H, et al. (2020) Research the effects of different fermentation times of yeast fermentation[J]Yunnan Chemical Technology. (in Chinese)

15. Bao W, Han J, Zhang Z, et al. (2021) Analysis of microbial community diversity in traditional fermented vegetables in Zhejiang using high-throughput sequencing[J].Food Science. https//:doi. 10.7506/spkx1002-6630-20191205-061. (in Chinese)

16. Torija M J, Rozès N, Poblet M, et al. (2001) Yeast population dynamics in spontaneous fermentations: Comparison between two different wine-producing areas over a period of three years[J]. Antonie Van Leeuwenhoek. https//:doi. 10.1023/A:1012027718701.

17. Constanti M, Poblet M, Arola L, et al. (1997) Analysis of yeast populations during alcoholic fermentation in a newly established winery[J]. American Journal Of Enology And Viticulture. https//:doi. 10.1007/s001220050413.

18. Liu X Z, Sang M, Zhang X A, et al. (2017) Enhancing expression of SSU1 genes in Saccharomyces uvarum leads to sulfite tolerance increase and transcriptome profiles change. FEMS Yeast Research. https://doi.org/10.1093/femsyr/fox023.

19. White T J, Bruns T, Lee S, et al. (1990) In PCR protocols: a guide to methods and applications, ed. Innis M A, Gelfand D H, Sninsky J J, et al. Academic Press, San Diego, pp. 315-322.

20. Martin M. (2011) Cutadapt removes adapter sequences from high-throughput sequencing reads[J]. Embnet Journal. DOI: https://doi.org/10.14806/ej.17.1.200.

21. Rognes T, Flouri T, Nichols B, Quince C, Mahé F. (2016) VSEARCH: a versatile open source tool for metagenomics. PeerJ. DOI: 10.7717/peerj.2584.

22. Callahan B, McMurdie P \& Holmes S. (2017) Exact sequence variants should replace operational taxonomic units in marker-gene data analysis. The ISME Journal. https://doi.org/10.1038/ismej.2017.119.

23. Elolimy A, Alharthi A, Zeineldin M, et al. (2020) Residual feed intake divergence during the preweaning period is associated with unique hindgut microbiome and metabolome profiles in neonatal Holstein heifer calves[J]. Journal of Animal Science and Biotechnology. https//:doi.10.1186/s40104-019-0406-x.

24. Li H Y, Wang H, Wang H T, et al. (2020) Correction to: The chemodiversity of paddy soil dissolved organic matter correlates with microbial community at continental scales[J]. Microbiome. https//:doi.10.1186/s40168-020-00945-3.

25. Ondov B D, Bergman N H, Phillippy A M. (2011) Interactive metagenomic visualization in a Web browser[J]. BMC Bioinformatics. https//:doi.10.1186/1471-2105-12-385.

26. Chao A. (1984) Nonparametric estimation of the number of classes in a population[J]. Scandinavian Journal of Statistics. (in Chinese).

27. Good, I J. (1953) The population frequencies of species and the estimation of population parameters [J]. Biometrika. https//:doi.10.1186/1471-2105-12-385. 
28. Liu K, Zhang Y, Li Q, et al. (2020) Ethnic differences shape the alpha but not beta diversity of gut microbiota from school children in the absence of environmental differences. Microorganisms. 2020 Feb 14;8(2):254. doi: 10.3390/microorganisms8020254.

29. Pielou E C J. (1966) The Measurement of diversity in different types of biological collections[J]. Journal of Theoretical Biology. https//:doi.10.1016/0022-5193(66)90013-0.

30. Shannon C E. (1948) A mathematical theory of communication[J]. Bell Systems Technical Journal. https//:doi. 10.1002/j.1538-7305.1948.tb01338.x

31. Simpson E H. (1949) Measurement of diversity[J]. Nature. https//:doi. 10.1136/thx.27.2.261

32. Chao A, Ricotta C. (2019) Quantifying evenness and linking it to diversity, beta diversity, and similarity. Ecology.100(12):e02852. doi: 10.1002/ecy.2852.

33. Stout MJ, Zhou Y, Wylie KM, et al. (2017) Early pregnancy vaginal microbiome trends and preterm birth. American Journal of Obstetrics and Gynecology. doi: 10.1016/j.ajog.2017.05.030.

34. Lozupone CA, Hamady M, Kelley ST, et al. (2007) Quantitative and qualitative beta diversity measures lead to different insights into factors that structure microbial communities. Applied and Environmental Microbiology. doi: 10.1128/AEM.01996-06.

35. Bolyen E, Rideout JR, Dillon MR, et al. (2019) Author Correction: Reproducible, interactive, scalable and extensible microbiome data science using QIIME 2.[J] Nature Biotechnology. https://doi.org/10.1038/s41587-019-0209-9.

36. Wang D, Tang Y, Chen G, et al. (2020) Dynamic analysis of volatile components of salted radish during different fermentation processes[J]. Food Science. https//:doi. 10.7506/spkx1002-663020190414-186.

37. Zhang G, Li Z, Deng S, et al. (2021) Characterization and succession analysis of bacterial community diversity in different fermentation cycles of Hainan H382 cigar leaf[J]. Acta Tabacaria Sinica. (in Chinese).

38. Fleet G H. (2008) Wine yeasts for the future[J]. FEMS Yeast Research. https//:doi.10.1111/j.15671364.2008.00427.x.

39. Delgado-Baquerizo M, Maestre F T, Reich Peter B, et al. (2016) Microbial diversity drives multifunctionality in terrestrial ecosystems[J]. Nature Communications. https//:doi. 10.1038/ncomms10541.

40. Wu X, Liu Q, Zhang H, et al. (2020) Research the effects of different fermentation times of yeast fermentation[J]Yunnan Chemical Technology. (in Chinese).

41. Liu Q, Wu Z, Fan S, et al. (2016) A primary study on the factors affecting yeast growth in a mixed culture[J]. Modern Food Science \& Technology. https//:doi.10.13982/j.mfst.1673-9078.2016.7.028. (in Chinese).

42. Ding Y, Tao S, Ma J, et al. (2018) Screening of the best medium of Yds-10 yeast[J]. Journal of Jiamusi University (Natural Science Edition). https//:doi. 10.3969/j.issn.1008-1402.2018.06. 027. (in Chinese). 
43. Čadež N, Pagnocca FC, Raspor P et al. (2014) Hanseniaspora nectarophila sp. nov., a yeast species isolated from ephemeral flowers[J]. International Journal of Systematic and Evolutionary Microbiology. https//:doi. 10.1099/ijs.0.061499-0.

44. Wang Y, Zhao Y C, Fan L L, et al. (2018) Identification and characterization of Pichia membranifaciens $\mathrm{Hmp}-1$ isolated from spoilage blackberry wine[J]. Journal of Integrative Agriculture. https//:doi. 10.1016/S2095-3119(18)62027-1.

45. Tan Y, Hao N. (2020) Effect of Mixed fermentation of Saccharomyces cerevisiae and NonSaccharomyces cerevisiae on fruit wine quality[J]. Science and Technology of Food Industry. https//:doi. 10.13386/j.issn1002-0306.2020.08.057. (in Chinese)

46. Čadež N, Pagnocca FC, Raspor P et al. (2014) Hanseniaspora nectarophila sp. nov., a yeast species isolated from ephemeral flowers[J]. International Journal of Systematic and Evolutionary Microbiology. https//:doi. 10.1099/ijs.0.061499-0.

47. Hofmeyer T, Hackenschmidt S, Nadler F, et al. (2016) Draft genome sequence of Cutaneotrichosporon curvatus DSM 101032 (Formerly Cryptococcus curvatus), an oleaginous yeast producing polyunsaturated fatty acids[J]. Genome Announcements. https//:doi.10.1128/genomeA.00362-16.

48. Gong Z, Shen H, Zhou W, Wang Y, Yang X, Zhao ZK. (2015) Efficient conversion of acetate into lipids by the oleaginous yeast Cryptococcus curvatus. Biotechnol Biofuels. https//:doi. 10.1186/s13068015-0371-3. eCollection 2015.

49. Brežná B, Zenišová K, Chovanová K, Chebeňová V, Kraková L, Kuchta T, Pangallo D. Evaluation of fungal and yeast diversity in Slovakian wine-related microbial communities. Antonie Van Leeuwenhoek. 2010 Nov;98(4):519-29. https//:doi. 10.1007/s10482-010-9469-6.

50. Park KH, Liu Z, Park CS, et al. (2016) Microbiota associated with the starter cultures and brewing process of traditional Hong Qu glutinous rice wine. Food Science and Biotechnology. https//:doi. 10.1007/s10068-016-0115-6.

51. Xie J, Ye Y, Wu Z, et al. (2021) Screening of key fungal strains in the fermentation process of the Chinese medicinal preparation "Lianzhifan Solution" based on metabolic profiling and highthroughput sequencing technology. Frontiers in Microbiology. https//:doi. 10.3389/fmicb.2021.727968.

52. Medina K, Boido E, Fariña L, et al. (2016) Non-Saccharomyces and Saccharomyces strains cofermentation increases acetaldehyde accumulation: effect on anthocyanin-derived pigments in Tannat red wines[J]. Yeast. https//:doi.10.1002/yea.3156.

53. Zhan JC, Cao MZ, You YL, et al. Research advance on the application of Non-Saccharomyces in winemaking. Scientia Agricultura Sinica. https//:doi.10.3864/j.issn.0578-1752.2020.19.018.

54. Guarcello R, Gaglio R, Todaro A, et al. (2019) Insights into the cultivable microbial ecology of "Manna" ash products extracted from Fraxinus angustifolia (Oleaceae) trees in Sicily, Italy. Frontiers in Microbiology.https//:doi.10.3389/fmicb.2019.00984. 
55. Batista NN, Ramos CL, Ribeiro DR, et al. (2015) Dynamic behavior of Saccharomyces cerevisiae, Pichia kluyveri and Hanseniaspora uvarum during spontaneous and inoculated cocoa fermentations and their effect on sensory characteristics of chocolate[J]. LWT-Food Science \& Technology. https//:doi. 10.1002/yea.3156.

56. Anfang N, Brajkovich M, Goddard M R. (2009) Co-fermentation with Pichia Kluyveri increases varietal thiol concentrations in Sauvignon Blanc[J]. Australian Journal of Grape and Wine Research. DOI: 10.1111/j.1755-0238.2008.00031.x

57. Ciani M, Pi Cc, lotti G. (1995) The growth kinetics and fermentation behavior of some nonSaccharomyces yeast associated with wine-making[J]. Biotechnology Letters. https//:doi. 10.1002/yea.3156.

\section{Tables}

Table 1 Yeast diversity indexes.

\begin{tabular}{|lllllll|}
\hline Sample & Chao1 & Good's coverage & Observed species & $\begin{array}{l}\text { Pielou's } \\
\text { evenness }\end{array}$ & Shannon & Simpson \\
\hline D1P & 24.00 & 1.00 & 90.57 & 0.65 & 4.20 & 0.90 \\
\hline D3P & 16.81 & 1.00 & 59.83 & 0.47 & 2.75 & 0.76 \\
\hline D5P & 14.75 & 1.00 & 40.37 & 0.36 & 1.90 & 0.55 \\
\hline D15P & 15.04 & 1.00 & 43.83 & 0.44 & 2.40 & 0.70 \\
\hline CK & 23.96 & 1.00 & 71.57 & 0.26 & 1.59 & 0.42 \\
\hline
\end{tabular}

\section{Figures}

\section{Figure 1}

Tree circle accumulation of fungal classification.

Note: The largest circle in the figure represented the level of the gate, while the gradually shrinking circles represented the class, order, family, genus, and species according to the gradient. The innermost dot represented the top 100 abundance OTUs. Its size (area) was directly a proportion of the abundance of the OTUs. The areas of the origin within the circles represented the abundance of the taxa corresponding to the circle. Each pie chart showed the composition ratio of the OTUs in each group. The larger the sector area, the higher the abundance of the taxon in the corresponding group was. 


\section{Figure 2}

Krona diagram of sample species composition.

Note: The krona circle diagram represented the seven taxonomic levels of a domain, phylum, class, order, family, and species from the inside to outside. The sizes of the sectors reflected the relative abundance of different taxa and had specific values. The different colors represented different taxa.

\section{Figure 3}

Histogram of the species composition of each sample.

Note: The abscissa was the sample name of the grouping scheme, and the ordinate was the relative abundance of each taxon at a specific classification level. Genera were distinguished by different colors.

\section{Figure 4}

UPGMA cluster analysis of the species in the samples.

Note: The UPGMA cluster was conducted according to the Euclidean distances. The vertical axis on the right was the top ten species, and the abscissa was the sample number.

\section{Figure 5}

Sample fungal diversity index.

Note: Each panel corresponded to an alpha diversity index, marked by a gray area at the top. In each panel, the abscissa was the grouping label, and the ordinate was the value of the corresponding alpha diversity index. The meanings of each symbol in the box and line chart were as follows: upper and lower end lines of the box, upper and lower quartiles (Interquartile range, IQR). The median line represented the median, and the upper and lower edges represented maximum and minimum values (extreme values within 1.5 times the IQR range). Points outside the upper and lower edges indicated outliers.

\section{Figure 6}

Hierarchical cluster analysis diagram among samples of each group. 
Note: The panel on the left was a hierarchical clustering tree diagram, and the samples were clustered according to the similarity between each other. The shorter the branch length between the samples, the more similar the two samples were. The panel on the right (drawn by default) was the abundance ranking Stacked histogram of the top ten species.

\section{Supplementary Files}

This is a list of supplementary files associated with this preprint. Click to download.

- SupplementalTable1.xlsx

- SupplementalTable2.xlsx

- SupplementalTable3.xlsx

- SupplementalTable4.xlsx

- SupplementaryFig1.tif

- SupplementaryFig2.tif

- SupplementaryFig3.tif

- SupplementaryFig4a.tif

- SupplementaryFig5a.tif 